\title{
Localização de centros de auxílio e distribuição de suprimentos em operações de resposta a desastres
}

\author{
Alfredo Moreno ${ }^{1}$, Douglas Alem ${ }^{2}$, Deisemara Ferreira ${ }^{3}$ \\ 1Departamento de Engenharia de Produção, UFSCAR-Sorocaba, SP, Brasil, alfredmorenoarteaga@gmail.com \\ 2Departamento de Engenharia de Produção, UFSCAR-Sorocaba, SP, Brasil, douglas@ufscar.br \\ ${ }^{3}$ Departamento de Física Química e Matemática, UFSCAR-Sorocaba, SP, Brasil, deisemaraferreira@gmail.com
}

\section{Recebido:}

6 de junho de 2017

Aceito para publicação:

13 de julho de 2017

Publicado:

31 de agosto de 2017

Editor de área:

Renato Lima, UNIFEI

\section{Palavras-chaves:}

Desastres naturais.

Logística humanitária.

Modelagel matemática.

Programação estocástica.

\section{Keywords:}

Natural disasters.

Humanitarian logistics.

Mathematical modeling.

Stochastic programming.

DOI:10.14295/transportes.v25i2.1168

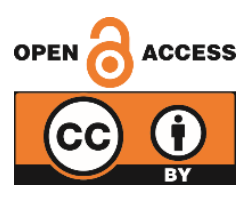

\begin{abstract}
RESUMO
A localização de centros de auxílio para o atendimento das vítimas e a distribuição de suprimentos essências à sobrevivência são operações chaves em situações de desastre. Embora muitos trabalhos da literatura tenham desenvolvidos modelos matemáticos para auxiliar em alguma dessas decisões, poucos autores se preocuparam em integrar ambas as decisões com o dimensionamento da frota na tentativa de gerar soluções mais eficientes. Nesse artigo são desenvolvidos dois modelos teóricos de programação estocástica inteira mista para apoiar as decisões de localização, distribuição e dimensionamento da frota de forma integrada minimizando o custo total esperado. Para resolver os modelos, foram exploradas variações de métodos híbridos que combinam métodos exatos e heurísticos implementados no solver CPLEX. Os modelos matemáticos foram analisados com base nas informações do megadesastre da região Serrana do Rio de Janeiro de 2011. Os resultados indicam que a configuração adequada do método híbrido pode retornar soluções com até $34 \%$ de melhoria em relação à opção padrão. Os modelos mostraram ser potencialmente úteis para o apoio às decisões dos órgãos encarregados das ações de resposta a desastres.
\end{abstract}

\section{ABSTRACT}

The location of relief centers and the distribution of vital supplies are important operations in disaster situations. Many works in the literature have developed mathematical models to assist in some of these decisions; however, few authors have integrated both decisions with fleet sizing in an attempt to generate solutions that are more efficient. In this paper, two stochastic programing models for the integrated problem of locationdistribution and fleet sizing are developed to minimize the total expected cost. To solve the models, different configuration of the hybrid and exact methods in the CPLEX solver were tested. The models were analyzed based on information from the megadisaster in the Mountain Region of the State of Rio de Janeiro in January 2011. Results show that the modification of the parameters of hybrid methods of CPLEX can improve by around $34 \%$ the cost of solutions in relation to the solution of the standard configuration. In addition, the models seem to be potentially useful to support decision of the bodies involved in response operations.

\section{INTRODUÇÃO}

A cada ano, centenas de desastres (terremotos, enchentes, furacões, vazamentos químicos, etc.) ocorrem no mundo, deixando milhares de vítimas fatais e milhões de afetados. Segundo dados do Emergency Events Database (EM-DAT, 2016), os últimos 30 anos contabilizaram, aproximadamente, 17.800 desastres ao redor do mundo, totalizando mais de 3 milhões de vítimas fatais, 6,3 bilhões de afetados e US \$2,7 bilhões de dólares em danos econômicos. No mesmo período no Brasil, houve 288 desastres (aproximadamente 1,6\% dos desastres mundiais), com 9.266 vítimas fatais, 57,3 milhões de pessoas afetadas e US \$15,7 milhões de dólares em danos econômicos. Frente a esses valores, as organizações governamentais e não governamentais precisam 
se preocupar em mitigar o impacto dos desastres sobre as populações vulneráveis. No contexto Brasileiro, os órgãos do Sistema Nacional de Defesa Civil são os responsáveis pela gestão de emergência em situações de desastres. Porém, os danos sociais e econômicos causados por esses eventos nos últimos anos têm evidenciado a dificuldade de tais organizações em responder eficientemente diante do impacto do desastre. No desastre da região Serrana do Rio de Janeiro em 2011, por exemplo, não existiam planos de contingências bem estruturados e socializados com a população, não houve coordenação dos diferentes atores envolvidos na fase inicial de resposta, e em alguns casos, a imprensa chegou antes das agências de ajuda humanitária em lugares considerados inacessíveis pela defesa civil (Busch e Amorim, 2011).

0 ciclo de vida das operações de desastre está ligado à temporalidade desse tipo de evento. Existem operações realizadas antes da ocorrência do desastre (pré-desastre) e operações realizadas após o início do desastre (pós-desastre). As decisões em operações pré-desastre incluem a localização de facilidades, o pré-posicionamento de estoque, o dimensionamento de frota (quantidade de veículos necessária para responder a qualquer que seja a consequência do desastre), o desenho e a socialização de planos de emergência, a proteção da infraestrutura, entre outras. As decisões em operações pós-desastre incluem localização de facilidades provisórias (abrigos), dimensionamento de frota (quantidade de veículos efetivamente utilizados na distribuição de produtos emergenciais e resgate, etc.), definição de níveis de estoques, transporte/distribuição de suprimentos, implementação de planos de emergência, resgate e evacuação de afetados, entre outras.

$\mathrm{Na}$ literatura científica encontramos diversos trabalhos que estudam as questões e dificuldades relativas às decisões de localização de centros de auxílio e também as decisões de distribuição/transporte. A integração das decisões de localização e distribuição/transporte compõe uma importante área de estudo em logística (Shen, 2007), que tem atraído a atenção de acadêmicos e tomadores de decisões. Como é de se esperar essas decisões estão relacionadas. Descentralizar uma quantidade maior de facilidades ao longo da rede, por exemplo, na tentativa de conseguir melhores rotas, pode reduzir o custo variável de transporte, mas, por outro, pode elevar os custos fixos de abertura e operação das facilidades. Centralizar as facilidades pode implicar na redução dos custos fixos de abertura dos centros de auxílio, no entanto, poderá gerar um aumento no custo de transporte do centro de auxílio até a área afetada. Esse é o tradeoff da logística tradicional, entre localização e transporte em termos do custo total da rede. Na logística humanitária (Apte, 2009), esse trade-off é ainda mais complexo, pois não necessariamente existe coordenação entre os diversos atores da rede humanitária para que a descentralização seja efetiva na redução do tempo de resposta dos planos emergenciais. Outro agravante é que, em muitas situações, a rede está colapsada e muitas rotas podem estar bloqueadas para alguns tipos de transporte, fazendo com que a decisão de centralizar as facilidades emergenciais dificulte ainda mais o acesso às vítimas, especialmente àquelas localizadas em lugares isolados. Em casos onde o número de veículos é escasso, a obtenção de planos viáveis na prática a partir da tomada de decisões de forma desagregada ainda mais difícil (Moreno et al., 2015). Tendo em vista a rede colapsada a seleção dos "melhores" tipos de transporte para cada tipo (ou impacto) de desastre é afeta o nível de serviço, ou seja, atendimento às vítimas. Do ponto de vista de custos, se, por um lado, uma frota superestimada gera custos excessivos e pode, portanto, ser inviável na prática, por outro lado, uma frota subestimada pode causar uma distribuição ineficiente de produtos, resultando em dificuldades adicionais em manter a equidade da distribuição entre as diversas áreas afetadas podendo então ocasionar o não atendimento da demanda de determinados itens. 
Pelas razões citadas acima, a integração das decisões de localização dos centros de auxílio com as decisões de distribuição/transporte no caso da logística humanitária é essencial, pois, além de favorecer a obtenção de planos viáveis de auxílio às vítimas ela, ao avaliar o trade off entre as decisões, retorna planos eficientes.

A dificuldade em antecipar o desastre e o seu impacto dificulta ainda mais a tomada de decisões. Com recursos limitados, os órgãos que fazem a gestão de desastres devem responder da melhor maneira possível a qualquer que seja a consequência desses eventos. Isso sugere que o desenvolvimento de ferramentas de apoio às decisões em desastres considere as incertezas que são inerentes a essas situações. A programação estocástica de dois estágios surge como uma maneira "natural" para modelar e resolver problemas em logística humanitária, sob a motivação de que os estágios podem ser relacionados ao ciclo de vida dos desastres (Mete e Zabinsky, 2010). 0 primeiro estágio pode ser visto como a fase de preparação, em que potenciais centros de auxílio, depósitos e veículos são selecionados, independentemente da realização das variáveis aleatórias. No segundo estágio, são determinadas as decisões de resposta ao desastre, i.e, as rotas que serão percorridas, quantos veículos serão, de fato, necessários e a efetiva distribuição de produtos.

Nesse artigo, são desenvolvidos dois modelos de programação estocástica de dois estágios com recurso para o problema integrado de localização, distribuição e dimensionamento de frota, em um contexto multi-período, multi-produto e multi-modal, e ainda considerando incertezas inerentes às situações de desastres. Os modelos diferenciam-se de outros da literatura pelo tratamento das incertezas, pela presença de um custo fixo associado à contratação de veículos e pela possibilidade de reutilizar os veículos para percorrer outras rotas dentro do mesmo período de tempo. Além disso, são explorados métodos de solução híbridos e exatos baseados em alterações do método branch-and-cut do solver CPLEX 12.5.1. 0 objetivo de tais alterações não é encontrar a melhor configuração do solver CPLEX dentro de todas as configurações possíveis, mas sim analisar algumas configurações alternativas mais eficientes.

Os modelos são analisados com base em instâncias geradas a partir de dados reais do megadesastre da Região Serrana do Rio de Janeiro em 2011. Este tipo de desastre é de grande representatividade no contexto nacional e internacional e tem sido pouco tratado por autores na literatura. Os modelos propostos são teóricos e, portanto, fornecem soluções que para serem aplicadas no contexto prático devem ser ajustadas às necessidades reais, combinadas com ferramentas de informação geográfica e implementadas com o apoio de especialistas e dos órgãos encarregados da gestão de desastres.

0 restante desse artigo está organizado da seguinte maneira. A Seção 2 apresenta a revisão bibliográfica. A Seção 3 descreve o problema e desenvolve os modelos matemáticos. A Seção 4 explora os métodos de solução do solver CPLEX. A Seção 5 discute os resultados computacionais. A Seção 6 apresenta as considerações finais e os direcionamentos da pesquisa.

\section{REVISÃO BIBLIOGRÁFICA}

Existem diversos trabalhos da literatura científica que mostram a evolução da aplicação de técnicas e modelos matemáticos no campo da logística humanitária. Altay e Green (2006) e Galindo e Batta (2013) revisaram as características dos principais trabalhos publicados na área de pesquisa operacional relacionados à gestão de desastres, identificando suas principais contribuições, os problemas que têm sido negligenciados e o potencial de estudo nessa área. Similar- 
mente, Ortuño et al. (2013) realizaram uma revisão dos modelos matemáticos utilizados na tomada de decisões de respostas a desastres. Grass e Fischer (2016) revisaram os modelos estocásticos de dois estágios aplicados à gestão de desastres, destacando os principais objetivos, supostos e características dos modelos.

Os poucos trabalhos encontrados na presente revisão bibliográfica que propuseram modelos determinísticos para a integração das decisões de localização, distribuição e dimensionamento da frota (Afshar et al., 2012; Lin et al., 2012; Nolz et al., 2010; Rath e Gutjahr, 2011; Song et al., 2009) basearam-se em um planejamento estático e/ou com frota homogênea. A principal limitação desses trabalhos é ignorar os custos associados à contratação ou utilização dos veículos e ignorar as incertezas inerentes às situações de desastres.

É notável a tendência de formulações de modelos que incorporam as incertezas via um conjunto finito de cenários no contexto da programação estocástica de dois estágios. Em geral, a demanda é a fonte de incerteza mais considerada nos trabalhos, mas a disponibilidade de arcos e de suprimentos, o tempo de transporte, alguns custos e a proporção de estoque utilizável também são fontes de incerteza comuns nos trabalhos analisados. A maioria dos modelos de programação estocástica apresentados na literatura apoiam as decisões de localização e pré-posicionamento de estoque no primeiro estágio e a distribuição de suprimentos no segundo estágio, mas sem considerar o dimensionamento da frota. Seguindo essa linha, tem-se Bozorgi-Amiri et al. (2011), Bozorgi-Amiri et al. (2013), Chang et al. (2007), Döyen et al. (2012), Li et al. (2011), Noyan (2012) e Rawls e Turnquist $(2010,2012)$. A principal limitação desses trabalhos é não integrar o problema de dimensionamento de frota ao problema de localização e distribuição de produtos.

Modelos matemáticos de programação estocástica que combinam decisões de localização, distribuição e dimensionamento da frota foram investigados em Mete e Zabinsky (2010) e Salmeron e Apte (2010), porém, em um contexto estático (mono-período) e sem a consideração de custos fixos associados à contratação de veículos para motivar a sua reutilização. Claramente, existe uma lacuna no estudo de modelos integrados em contextos dinâmicos (multi-período) e ainda considerando o tempo de transporte na distribuição de produtos, que é essencial em se tratando de horizontes de planejamento curtos. Nesse artigo, considera-se o problema de localização, distribuição e dimensionamento de forma integrada em um contexto multi-período, multi-produto e com frota heterogênea. Além disso, são consideradas como variáveis aleatórias a demanda, a proporção de estoque utilizável, os suprimentos e a disponibilidade das rotas, variáveis aleatórias que não têm sido estudadas em conjunto na literatura. Adicionalmente, poucos trabalhos têm estudado a logística humanitária em situações de inundações e movimentos de massas, desastres que têm causado grandes perdas nos últimos anos.

\section{DESCRIÇÃO DO PROBLEMA E MODELAGEM MATEMÁTICA}

O problema de localização e distribuição de suprimentos em operações de resposta a desastres proposto nesse trabalho combina quatro tipos de decisão: localização, dimensionamento de frota, distribuição e alocação de demanda.

O problema de localização de centros de auxílio determina em qual das possíveis localidades os centros de auxílio devem operar para o atendimento às vítimas. Eles possuem uma capacidade de armazenamento (volume) limitada que deve ser respeitada. A localização dos depósitos é conhecida, e assume-se que não estão limitados pela sua capacidade física, mas sim pela disponibilidade dos suprimentos. Podem existir estoques de produtos nos depósitos e nos centros 
de auxílio, sendo que nem toda a quantidade de produtos estocados nos centros de auxílio permanece em condições de ser utilizada de um período para o outro devido ao impacto do desastre. Devido o horizonte de planejamento ser curto, dias ou horas, assume-se também que os centros de auxílio abertos devem permanecer em funcionamento até o final das operações humanitárias.

O problema de dimensionamento da frota envolve a decisão de determinar a quantidade e os tipos de veículos que devem ser contratados em cada depósito para distribuir os produtos aos centros de auxílio. Os veículos são contratados por período e a sua contratação não implica que sejam de fato utilizados no transporte de produtos. 0 transporte de produtos, por sua vez, consiste em determinar a quantidade de cada tipo de produto que deve ser transportada dos depósitos até os centros de auxílio que armazenam temporariamente os produtos demandados pelas áreas afetadas. Além disso, deve-se determinar em que veículos devem transportados esses produtos, considerando que existem um conjunto de rotas que, como resultado do impacto do desastre, não estão disponíveis para alguns tipos de veículos. A quantidade de produtos que podem ser transportados está limitada pela capacidade dos veículos (em volume e peso).

O problema de alocação da demanda consiste em designar uma fração da demanda de cada área afetada aos centros de auxílio onde as vítimas podem se abastecer com os itens que necessitam. Assume-se um custo pelo atendimento da demanda que é proporcional à distância entre os centros de auxílio e as áreas afetadas.

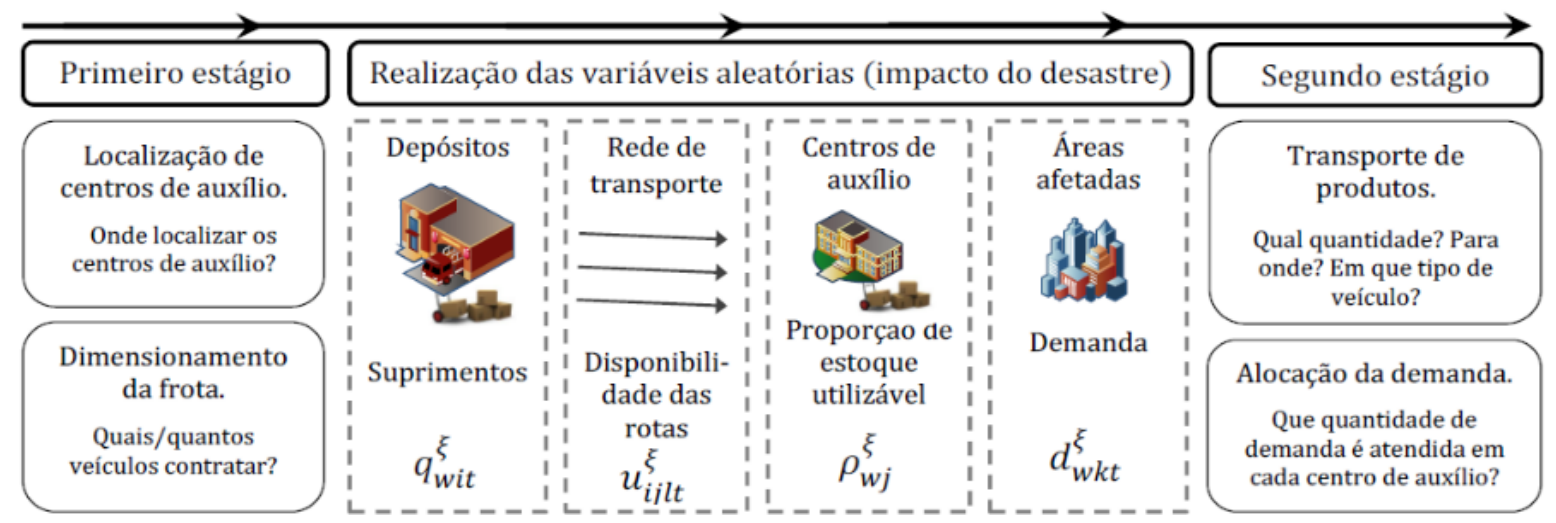

Figura 1. Decisões e parâmetros estocásticos no problema de distribuição-localização com dimensionamento da frota

Dado que não é possível conhecer com antecedência as consequências do impacto do desastre, foram assumidas as seguintes variáveis aleatórias: o suprimento de produtos nos depósitos, que é resultado de doações ou apoio econômico do governo; a proporção de estoque utilizável nos centros de auxílio, que é relativa às condições dos produtos transportados e às condições de armazenamento e ao impacto do desastre; a demanda de produtos nas áreas afetadas, que depende do impacto do desastre sobre a população; e a disponibilidade das rotas, que depende das consequências do desastre sobre a rede de transporte. As variáveis aleatórias são aproximadas por um conjunto discreto de realizações ou cenários, representado por $\Xi$, com probabilidades de ocorrência conhecidas $\pi(\xi)$ que designam a chance de ocorrência de tal cenário. 0 objetivo do modelo é realizar o atendimento das vítimas do desastre minimizando o custo total esperado. A Figura 1 representa as variáveis aleatórias envolvidas no problema, e as decisões de primeiro e segundo estágio. 


\subsection{Modelo 1: Modelo de localização-distribuição com decisões de frota}

Nesse primeiro modelo, a cada veículo é designado uma única viagem entre depósitos e centros de auxílio. Não se considera o tempo de transporte entre os nós. A notação matemática é apresentada a seguir.

\section{Conjuntos}

$\begin{array}{ll}\text { W } & \text { Produtos. } \\ \text { I } & \text { Depósitos. } \\ \text { J } & \text { Centros de auxílio. } \\ \text { K } & \text { Áreas afetadas (cidades ou regiões). } \\ \text { L } & \text { Tipos de veículos. } \\ \text { T } & \text { Períodos de tempo. } \\ \Xi & \text { Possíveis cenários de ocorrência das variáveis aleatórias. }\end{array}$

\section{Parâmetros determinísticos}

$\alpha_{j}^{\text {new }} \quad$ Custo fixo por abertura do centro de auxílio $j$ (infraestrutura básica requerida para começar as operações).

$\alpha_{j}^{\text {oper }} \quad$ Custo fixo por operação do centro de auxílio $j$ (energia elétrica, água, equipes de ajuda humanitária).

$\beta_{l} \quad$ Custo fixo por veículo tipo $l$ (compra, contratação ou manutenção).

$\gamma_{i j l} \quad$ Custo de transporte do veículo $l$ na rota $i \rightarrow j$.

$\varnothing_{w}^{+} \quad$ Custo de estoque do produto $w$.

$\varnothing_{w}^{-} \quad$ Penalidade por demanda insatisfeita do produto $w$.

$\delta_{k j} \quad$ Custo do centro de auxílio $j$ atender demanda da área afetada $k$.

$b_{w}\left(b_{w}^{\prime}\right)$ Volume (peso) do produto $w$.

$k_{l}^{v}\left(k_{l}^{\prime v}\right)$ Capacidade em volume (peso) do veículo $l$.

$k_{j}^{c} \quad$ Capacidade total do centro de auxílio $j$ (volume).

$k_{w j}^{c p} \quad$ Capacidade de armazenamento do produto $w$ no centro de auxílio $j$ (quantidade de produtos).

$k_{i j l}^{a r c} \quad$ Número máximo de veículos $l$ que podem atravessar a rota $i \rightarrow j$.

$k_{l}^{n} \quad$ Número máximo de veículos $l$ disponíveis.

\section{Parâmetros estocásticos}

$q_{\text {wit }}(\xi) \quad$ Suprimento do produto $w$ no depósito $i$ no período $t$ no cenário $\xi$.

$\rho_{w j t}(\xi) \quad$ Proporção do produto $w$ no centro de auxílio $j$ no cenário $\xi$ que permanece utilizável entre dois períodos $t-1$ e $t$. 
$d_{w k t}(\xi) \quad$ Demanda do produto $w$ na área afetada $k$ no período $t$ no cenário $\xi$.

$u_{i j l t}(\xi) \quad=1$ se a rota $i \rightarrow j$ está disponível para o veículo $l$ no período $t$ no cenário $\xi$. $=0$, caso contrario.

$\pi(\xi) \quad$ Probabilidade de ocorrência do cenário $\xi$.

Variáveis de decisão de primeiro estágio

$N_{\text {ilt }} \quad$ Número de veículos $l$ designados ao depósito $i$ no período $t$.

$Y_{j t}^{\text {new }} \quad=1$, se o centro de auxílio $j$ abre no período $t$

$=0$, caso contrario.

$Y_{j t}^{o p e r}=1$, se o centro de auxílio $j$ está em operação no período $t$

$=0$, caso contrario.

Variáveis de decisão de segundo estágio

$P_{\text {wijlt }}(\xi) \quad$ Quantidade do produto $w$ transportado na rota $i \rightarrow j$ pelo veículo $l$ no período $t$ no cenário $\xi$.

$V_{i j l t}(\xi) \quad$ Número de veículos $l$ usados na rota $i \rightarrow j$ no período $t$ no cenário $\xi$.

$z_{w j k t}(\xi) \quad$ Quantidade do produto $w$ no centro de auxílio $j$ para atender demanda da área afetada $k$ no período $t$ no cenário $\xi$.

$I_{w i t}^{d}(\xi) \quad$ Estoque do produto $w$ no depósito $i$ no período $t$ no cenário $\xi$.

$I_{w j t}^{r c}(\xi) \quad$ Estoque do produto $w$ no centro de auxílio $j$ no período $t$ no cenário $\xi$.

$U_{w k t}(\xi)$ Demanda insatisfeita do produto $w$ na área afetada $k$ no período $t$ no cenário $\xi$.

Seja $\Lambda$ conjunto de todas as variáveis de decisão. 0 modelo de programação estocástica inteira-mista de dois estágios para o problema de localização-distribuição de suprimentos com decisões de dimensionamento é formulado da seguinte forma:

$$
\min _{\Lambda} Y C^{\text {new }}+Y C^{\text {oper }}+N C+V C+Z C+I C+U C
$$

em que

$Y C^{\text {new }}=\sum_{j \in \mathrm{J}} \sum_{t \in \mathrm{T}} \alpha_{j}^{\text {new }} \cdot Y_{j t}^{\text {new }}$ é o custo total de abertura dos centros de auxílio;

$Y C^{\text {oper }}=\sum_{j \in \mathrm{J}} \sum_{t \in \mathrm{T}} \alpha_{j}^{\text {oper }} \cdot Y_{j t}^{\text {oper }}$ é o custo total de operação dos centros de auxílio;

$N C=\sum_{l \in \mathrm{L}} \sum_{i \in \mathrm{I}} \sum_{t \in \mathrm{T}} \beta_{l} \cdot N_{i l t}$ é o custo total pela designação (contratação) dos veículos;

$V C=\sum_{i \in \mathrm{I}} \sum_{j \in \mathrm{J}} \sum_{l \in \mathrm{L}} \sum_{t \in \mathrm{T}} \sum_{\xi \in \Xi} \pi(\xi) \cdot \gamma_{i j l} \cdot V_{i j l t}(\xi)$ é o custo total de transporte; 
$Z C=\sum_{w \in \mathrm{W}} \sum_{k \in \mathrm{K}} \sum_{j \in \mathrm{J}} \sum_{t \in \mathrm{T}} \sum_{\xi \in \Xi} \pi(\xi) \cdot \delta_{k j} \cdot z_{w j k t}(\xi)$ é o custo total de atendimento das área afetadas;

$I C=\sum_{w \in \mathrm{W}} \sum_{j \in \mathrm{J}} \sum_{t \in \mathrm{T}} \sum_{\xi \in \Xi} \pi(\xi) \cdot \varnothing_{w}^{+} \cdot I_{w j t}^{r c}(\xi)$ é o custo total de estoque nos centros de auxílio;

$U C=\sum_{w \in \mathrm{W}} \sum_{k \in \mathrm{K}} \sum_{t \in \mathrm{T}} \sum_{\xi \in \Xi} \pi(\xi) \cdot \varnothing_{w}^{-} \cdot U_{w k t}(\xi)$ é a penalidade total por demanda insatisfeita.

Sujeito às seguintes restrições:

$$
\begin{aligned}
& \sum_{i \in \mathrm{I}} \sum_{l \in \mathrm{L}} P_{w i j l t}(\xi)+\rho_{w j t}(\xi) \cdot I_{w j(t-1)}^{r c}(\xi)=\sum_{k \in \mathrm{K}} Z_{w k j t}(\xi)+I_{w j t}^{r c}(\xi), \forall w \in \mathrm{W}, j \in \mathrm{J}, t \in \mathrm{T}, \xi \in \Xi . \\
& q_{w i t}(\xi)+I_{w i(t-1)}^{d}(\xi)=\sum_{j \in \mathrm{J}} \sum_{l \in \mathrm{L}} P_{w i j l t}(\xi)+I_{w i t}^{d}(\xi), \forall w \in \mathrm{W}, i \in \mathrm{I}, \mathrm{t} \in \mathrm{T}, \xi \in \Xi . \\
& U_{w k t}(\xi)=d_{w k t}(\xi)-\sum_{j \in \mathrm{J}} Z_{w k j t}(\xi)+U_{w k(t-1)}(\xi), \forall w \in \mathrm{W}, k \in \mathrm{K}, \mathrm{t} \in \mathrm{T}, \xi \in \Xi . \\
& \sum_{i \in \mathrm{I}} \sum_{l \in \mathrm{L}} \sum_{w \in \mathrm{W}} b_{w} \cdot P_{w i j l t}(\xi)+\sum_{w \in \mathrm{W}} b_{w} \cdot I_{w j(t-1)}^{r c}(\xi) \leq k_{j}^{c} \cdot Y_{j t}^{\text {oper }}, \forall j \in \mathrm{J}, \mathrm{t} \in \mathrm{T}, \xi \in \Xi . \\
& \sum_{i \in \mathrm{I}} \sum_{l \in \mathrm{L}} P_{w i j l t}(\xi)+I_{w j(t-1)}^{r c}(\xi) \leq k_{w j}^{c p} \cdot Y_{j t}^{o p e r}, \forall w \in \mathrm{W}, j \in \mathrm{J}, \mathrm{t} \in \mathrm{T}, \xi \in \Xi . \\
& V_{i j l t}(\xi) \geq \frac{\sum_{w \in \mathrm{W}} b_{w} \cdot P_{w i j l t}(\xi)}{k_{l}^{v}}, \forall i \in \mathrm{I}, j \in \mathrm{J}, l \in \mathrm{L}, \mathrm{t} \in \mathrm{T}, \xi \in \Xi . \\
& V_{i j l t}(\xi) \geq \frac{\sum_{w \in \mathrm{W}} b_{w}^{\prime} \cdot P_{w i j l t}(\xi)}{k_{l}^{\prime v}}, \forall i \in \mathrm{I}, j \in \mathrm{J}, l \in \mathrm{L}, \mathrm{t} \in \mathrm{T}, \xi \in \Xi . \\
& V_{i j l t}(\xi) \geq k_{i j l}^{a r c} \cdot u_{i j l t}(\xi), \forall i \in \mathrm{I}, j \in \mathrm{J}, l \in \mathrm{L}, \mathrm{t} \in \mathrm{T}, \xi \in \Xi \text {. } \\
& \sum_{i \in \mathrm{I}} N_{i l t} \leq k_{l}^{n}, \forall l \in \mathrm{L}, \mathrm{t} \in \mathrm{T} . \\
& \sum_{j \in \mathrm{J}} V_{i j l t}(\xi) \leq N_{i l t}, \forall i \in \mathrm{I}, l \in \mathrm{L}, \mathrm{t} \in \mathrm{T}, \xi \in \Xi . \\
& Y_{j t}^{\text {oper }} \geq Y_{j(t-1)}^{\text {oper }}, \forall j \in \mathrm{J}, \mathrm{t} \in \mathrm{T} . \\
& Y_{j t}^{\text {new }} \geq Y_{j t}^{\text {oper }}-Y_{j(t-1)}^{\text {oper }}, \forall j \in \mathrm{J}, \mathrm{t} \in \mathrm{T} \text {. } \\
& \mathbf{Y}^{\text {oper }} \in \mathbb{B}^{|\mathrm{J}| \times|\mathrm{T}|} . \\
& \mathbf{Y}^{\text {new }} \geq 0 \text {. } \\
& \mathbf{N} \geq 0 \text {. } \\
& \mathbf{V}(\xi) \geq 0 \text {, e inteiro, } \forall \xi \in \Xi . \\
& \mathbf{P}(\xi), \mathbf{Z}(\xi), \mathbf{I}^{\mathrm{rc}}(\xi), \mathbf{U}(\xi), \mathbf{I}^{\mathrm{d}}(\xi) \geq 0 \text {. }
\end{aligned}
$$

A função objetivo (1) minimiza o custo total esperado. As restrições (2) e (3) garantem a conservação do fluxo de produtos nos centros de auxílio e nos depósitos, respectivamente. Na restrição (2), o estoque que pode ser utilizado de um período para o seguinte é reduzido pela 
proporção de estoque utilizável $\rho_{w j t}(\xi)$. A restrição (4) determina a demanda insatisfeita das áreas afetadas. Sem perda de generalidade, assume-se que os estoques e atraso iniciais são nulos. As restrições (5) e (6) garantem que não pode existir transporte em uma rota $i \rightarrow j$ se o centro de auxílio $j$ não está em operação. Se o centro de auxílio $j$ está em operação, essas duas restrições limitam o fluxo de produtos de acordo com a sua capacidade total e por produto. A capacidade por produto índica que os centros de auxílio podem não ter as condições necessárias para manter alguns produtos. As restrições (7) e (8) determinam o número mínimo de veículos necessários para realizar o transporte de produtos dos depósitos aos centros de auxílio no segundo estágio, respeitando as capacidades (peso e volume) dos veículos. A restrição (9) garante que o veículo $l$ só pode percorrer a rota $i \rightarrow j$ no período $t$ no cenário $\xi$ se a rota estiver disponível, i.e., $u_{i j l t}(\xi)=1$. Está restrição também limita o número de veículos que podem ser utilizados à capacidade de transporte na rota $k_{i j l}^{a r c}$. A restrição (10) restringe o número de veículos de cada tipo que podem ser contratados por período no primeiro estágio. A restrição (11) assegura que somente os veículos contratados no primeiro estágio podem fazer o transporte no segundo estágio. A restrição (12) garante que os centros de auxílio abertos devem permanecer em operação até o final das operações humanitárias. A restrição (13) associa as variáveis de decisão de abertura com aquelas referentes à operação dos mesmos. As restrições (14)(18) são os domínios das variáveis de decisão.

\subsection{Modelo 2: Modelo de localização-distribuição com decisões de frota e reutilização de veículos}

Diferente do modelo anterior, o modelo 2 considera a possibilidade da reutilização de veículos dentro de um mesmo período, o que permite reduzir o custo fixo total de contratação dos veículos. Para definir o modelo 2, são considerados os parâmetros e variáveis do modelo anterior e a seguinte notação matemática adicional:

\section{Conjuntos}

L' Conjunto dos veículos disponíveis.

\section{Parâmetros determinísticos}

$\tau_{i j l} \quad$ Tempo de transporte do veículo $l$ na rota $i \rightarrow j$.

$\ell_{t} \quad$ Tamanho do período $t$.

$M \quad$ Número suficientemente grande, pode ser estabelecido como $\ell_{t}$.

Variáveis de decisão de primeiro estágio

$N^{\prime}{ }_{i l t} \quad=1$ se o veículo $l$ é designado no depósito $i$ no período $t$. $=0$, caso contrário.

Variáveis de decisão de segundo estágio $V_{i j t}^{\prime}(\xi)$ Número de vezes que o veículo $l$ percorre a rota $i \rightarrow j$ no período $t$ no cenário $\xi$. 
Note que o modelo 2 considera um novo conjunto L' de todos os possíveis veículos, enquanto que o modelo 1 considera o conjunto $L$ apenas dos tipos de veículos disponíveis. Para instâncias similares $\left|\mathrm{L}^{\prime}\right| \geq|\mathrm{L}|$. São consideradas duas novas variáveis, $N^{\prime}{ }_{i l t}$ e $V_{i j l t}^{\prime}(\xi)$, que substituem as variáveis $N_{i l t}$ e $V_{i j l t}(\xi)$, respectivamente. Por simplicidade, assume-se que o tempo de transporte nos arcos $i \rightarrow j$ e $j \rightarrow i$ são iguais. 0 parâmetro $\ell_{t}$ é expresso na mesma unidade do parâmetro $\tau_{i j l}$, e.g., para $\tau_{i j l}$ em blocos de duas horas, $\ell_{t}=12$ indica que o dia $t$ tem 12 blocos de duas horas. A função objetivo (19) diferencia-se da função objetivo (1) pelo conjunto L' no lugar do conjunto L. Além disso, foram substituídas as variáveis $N_{i l t}$ e $V_{i j l t}(\xi)$ por $N_{i l t}^{\prime}$ e $V_{i j l t}^{\prime}(\xi)$, respectivamente, como mostrado a seguir:

$$
\min _{\Lambda} Y C^{n e w}+Y C^{o p e r}+N C^{\prime}+V C^{\prime}+Z C+I C+U C
$$

em que

$$
\begin{array}{r}
N C^{\prime}=\sum_{l \in \mathrm{L}} \sum_{i \in \mathrm{I}} \sum_{t \in \mathrm{T}} \beta_{l} \cdot N^{\prime}{ }_{i l t} \text { é o custo total pela designação (contratação) dos veículos; } \\
V C^{\prime}=\sum_{i \in \mathrm{I}} \sum_{j \in \mathrm{J}} \sum_{l \in \mathrm{L}} \sum_{t \in \mathrm{T}} \sum_{\xi \in \Xi} \pi(\xi) \cdot \gamma_{i j l} \cdot V^{\prime}{ }_{i j l t}(\xi) \text { é o custo total de transporte. }
\end{array}
$$

As restrições (2), (3), (4), (5), (6), (13) e (14) diferenciam-se das correspondentes restrições do modelo 1 somente pela substituição do conjunto L' no lugar do conjunto L. As demais restrições foram modificadas conforme segue.

$$
\begin{gathered}
V_{i j l t}^{\prime}(\xi) \geq \frac{\sum_{w \in \mathrm{W}} b_{w} \cdot P_{w i j l t}(\xi)}{k_{l}^{v}}, \forall i \in \mathrm{I}, j \in \mathrm{J}, l \in \mathrm{L}, \mathrm{t} \in \mathrm{T}, \xi \in \Xi . \\
V_{i j l t}^{\prime}(\xi) \geq \frac{\sum_{w \in \mathrm{W}} b_{w}^{\prime} \cdot P_{w i j l t}(\xi)}{k_{l}^{\prime v}}, \forall i \in \mathrm{I}, j \in \mathrm{J}, l \in \mathrm{L}, \mathrm{t} \in \mathrm{T}, \xi \in \Xi . \\
V_{i j l t}^{\prime}(\xi) \leq M \cdot u_{i j l t}(\xi) \cdot N^{\prime}{ }_{i l t}, \quad \forall i \in \mathrm{I}, j \in \mathrm{J}, l \in \mathrm{L}^{\prime}, \mathrm{t} \in \mathrm{T}, \xi \in \Xi . \\
\sum_{j \in \mathrm{J}} 2 \cdot \tau_{i j l} \cdot V^{\prime}{ }_{i j l t}(\xi) \leq \ell_{t}, \quad \forall i \in \mathrm{I}, l \in \mathrm{L}^{\prime}, \mathrm{t} \in \mathrm{T}, \xi \in \Xi . \\
\mathbf{N}^{\prime} \in \mathbb{B}^{|\mathrm{I}||\times| \mathrm{L}|\times| \mathrm{T} \mid} . \\
\mathbf{V}^{\prime}(\xi) \geq 0, \text { inteiro, }, \forall \xi \in \Xi .
\end{gathered}
$$

As restrições (20) e (21) determinam o número mínimo de veículos necessários para realizar o transporte de produtos dos depósitos aos centros de auxílio no segundo estágio, respeitando as capacidades (peso e volume) dos veículos. A restrição (22) assegura que só os veículos contratados no primeiro estágio podem transportar produtos pelos arcos disponíveis no segundo estágio. Note que para existir transporte no segundo estágio, a rota deve estar disponível ( $u_{i j l t}(\xi)=1$ ) e o veículo deve ser contratado $\left(N^{\prime}{ }_{i l t}=1\right)$. A restrição (23) limita o tempo total de 
transporte dos veículos ao tamanho do período, i.e., a soma dos percursos dos veículos em um período tem que ser menor do que o tamanho do período. As restrições (24) e (25) representam o domínio das variáveis de decisão.

\section{ANÁLISE DE CONFIGURAÇÕES DO SOLVER CPLEX PARA RESOLVER OS MODELOS}

Alterações nos parâmetros do solver CPLEX foram realizadas para resolver as formulações matemáticas. 0 solver CPLEX é um dos pacotes de otimização mais eficientes do mercado. Possui implementados métodos exatos e heurísticos eficientes para solução de problemas gerais. No entanto, para problemas específicos e de grande porte como o caso dos modelos propostos neste artigo, sua utilização com configuração dos parâmetros no modo default não conseguiu resolver eficientemente a maioria das instâncias propostas. Sendo assim, foram exploradas estratégias alternativas à versão default com o intuito de diminuir o tempo computacional e o gap de otimalidade na solução das instâncias. De fato, é sabido que alterações de configuração de alguns parâmetros do solver podem representar melhorias no desempenho computacional (Hutter et al., 2009). Porém, a calibração de parâmetros pode ser uma tarefa complexa devido à natureza combinatória do número de possibilidades de combinações de parâmetros. Logo, o foco na presente pesquisa não é encontrar a melhor configuração dentro de todas as configurações possíveis, mas sim analisar algumas configurações alternativas mais eficientes. Essas configurações são baseadas na modificação da escolha de métodos de solução tanto exatos quanto heurísticos e suas combinações mais promissoras. Basicamente, foram testadas 15 configurações, a saber:

- CPX1: Default.

- CPX2: Desligar planos de corte.

- CPX3: Desligar pré-solver.

- CPX4: Desligar heurística RINS.

- CPX5: Utilizar heurística RINS a cada 100 nós.

- CPX6: CPX2 + CPX4.

- CPX7: СРX2 + СРX3.

- CPX8: CPX2 + utilizar a heurística RINS a cada 10 nós.

- CPX9: CPX2 + CPX5.

- CPX10: CPX2 + algoritmo primal simplex na relaxação e nos subproblemas nos nós.

- CPX11: CPX2 + algoritmo dual simplex na relaxação e nos subproblemas nos nós.

- CPX12: CPX2 + algoritmo network simplex na relaxação e nos subproblemas nos nós.

- CPX13: CPX2 + algoritmo barrier na relaxação e nos subproblemas nos nós.

- CPX14: Utilizar algoritmo dual simplex na relaxação e nos subproblemas nos nós.

- CPX15: CPX2 + CPX5 + CPX14.

Nos testes, o método de planos de corte, que implica na inclusão de outras equações/ inequações no modelo, foi desligado. A conjectura é que eles podem deixar o modelo ainda mais complexo com a inclusão de novas restrições (Ferreira et al., 2010) sem, entretanto, melhorar os limitantes e acelerar a convergência do método. A frequência de utilização (a cada quantos nós) da heurística RINS (Relaxation Induced Neighborhood Search), que explora a vizinhança da solução incumbente com o objetivo de melhorar a solução e diversificar a busca (Danna et al., 
2005), é definida automaticamente no solver CPLEX default. Não existe, a priori, uma frequência padrão de utilização desta heurística. Aplicar a heurística RINS em cada nó pode levar a subproblemas MIP similares sem, entretanto, obter melhorias. Por outro lado, aplicar a heurística RINS em intervalos maiores (em número de nós) pode reduzir o benefício da sua utilização. Nesse estudo, a frequência da sua utilização foi alterada para cada 10 e/ou 100 nós da árvore branch-and-bound. 0 pré-solver - que ajuda a simplificar o problema eliminando redundâncias (variáveis, restrições redundantes) - foi desligado, pois, em alguns casos, o problema reduzido é mais difícil de ser resolvido (ILOG, 2011). Como a relaxação linear dos modelos foi encontrada para instâncias grandes em até 3.600 segundos, foram testadas outras estratégias para resolver os LPs (problemas lineares) na tentativa de reduzir estes tempos computacionais. Foram analisados os algoritmos primal, dual e network simplex e o algoritmo de pontos interiores (Barrier) para resolver as relaxações lineares do nó raiz e também para resolver os subproblemas de cada nó da árvore.

\section{TESTES COMPUTACIONAIS}

O objetivo dessa seção é analisar o desempenho das estratégias de solução. Os modelos foram programados no software GAMS 24.1.3 e resolvidos pelo solver CPLEX 12.5.1 em um computador com 16 GB de memória RAM, processador Intel Core i7 e sistema operacional Windows 7. Foi estabelecido como critério de parada um limite de tempo de 3.600 segundos ou um gap de otimalidade de $1 \%\left(\mathrm{GAP}=\frac{\text { Solução MIP }- \text { Limitante inferior }}{\text { Solução MIP }}\right)$.

\subsection{Características das instâncias}

Incialmente foram geradas três instâncias (S1, M1 e L1) a partir dos dados estimados com base no megadesastre da região Serrana do Rio de Janeiro em 2011 (Rio de Janeiro, 2011).

A instância S1 considera 3 depósitos, 8 centros de auxílio, 5 cidades afetadas, 3 produtos (água, alimentos, produtos médicos), 2 tipos de veículos no conjunto L (10 caminhões e 5 barcos no conjunto L'), 3 cenários (emergência, crise, menor) e 5 períodos. A instância M1 considera 3 depósitos, 20 centros de auxílio, 5 cidades afetadas, 5 tipos de produtos (água, alimentos, produtos médicos, produtos de higiene doméstica, produtos de higiene pessoal), 3 tipos de veículos no conjunto L (35 caminhões, 15 barcos e 10 helicópteros no conjunto L'), 5 cenários (emergência, crise, menor, moderado, maior) e 10 períodos. A instância L1 considera 6 depósitos, 40 centros de auxílio, 10 cidades afetadas, 5 tipos de produtos, 3 tipos de veículos no conjunto L (35 caminhões, 15 barcos e 10 helicópteros no conjunto L'), 6 cenários (emergência, crise, menor, moderado, maior, catástrofe) e 20 períodos. No modelo 1 , o número de veículos considerados foi assumido grande o suficiente, enquanto que no modelo 2 está limitado pela cardinalidade do conjunto L'.

A partir dessas três instâncias, foram construídas 24 novas instâncias a partir de modificações em alguns parâmetros com o objetivo de analisar o comportamento do modelo em diversas situações. Foram exploradas situações em que o suprimento é alto (S2, M2, L2), a disponibilidades dos veículos é limitada (S3, M3, L3), um dos tipos de veículos não está disponível (S4, M4, L4), a capacidade dos centros de auxílio é reduzida (S5, M5, L5), a capacidade dos veículos é reduzida (S6, M6, L6), o custo de abertura dos centros de auxílio aumenta (S7, M7, L7), o tempo de transporte aumenta (S8, M8, L8) e o suprimento e a demanda diminuem (S9, M9, L9). As instâncias S8, M8 e L8 são consideradas somente para o modelo 2, pois esse modelo considera 
o tempo de transporte. Similarmente, as instâncias S3, M3 e L3 são somente consideradas para o modelo 1, dado que, para o modelo 2, essas instâncias são equivalentes às instâncias (S1, M1, L1).

\subsection{Análise dos Resultados via Perfis de desempenho}

Dois critérios foram utilizados para avaliar o comportamento dos métodos de solução: o tempo computacional medido em segundos, e a qualidade da resposta medida pelo gap de otimalidade. Para determinar a melhor estratégia, utilizou-se a ideia de perfis de desempenho de Dolan e Moré (2002). Os perfis de desempenho baseiam-se na função de distribuição acumulada $P(f, q): \mathbb{R} \rightarrow[0,1]$, que indica a probabilidade da estratégia $f$ estar dentro de um fator $q \in \mathbb{R}$ da melhor razão possível. A função $P(f, q)$ é definida por:

$$
P(f, q)=\frac{\left|\left\{p \epsilon P: \log _{2}(v(p, f)) \leq q\right\}\right|}{n_{p}}, q \geq 0,
$$

com,

$$
v(f, q)=\frac{T C_{p f}}{\min \left\{T C_{p f}: f \in F\right\}},
$$

em que $P$ é o conjunto de instâncias, $\operatorname{com} p=1, \ldots,|P| ; n_{p}=|P|$ é o número total de instâncias; $F$ é o conjunto de estratégias, $\operatorname{com} f=1, \ldots,|F| ; v(f, q)$ é a razão de melhoria definida como a razão entre $T C_{p f}$ que é o tempo (gap) quando a instância $p$ é resolvida com a estratégia $f$ e $\min \left\{T C_{p f}: f \in F\right\}$ que é o menor tempo (gap) em que a instância $p$ foi resolvida dentre todas as estratégias em $F$. A melhor estratégia é aquela que tenha o menor valor de $q$ para $P(f, q)=1$.

A Tabela 1 mostra os valores extremos da curva de perfis de desempenho para as estratégias CPLEX testadas com os modelos 1 e 2 . 0 valor de $P(f, q)$ quando $q=0$ indica a fração das instâncias para as quais a estratégia atingiu a melhor solução (em gap ou tempo). 0 valor de $q$ quando $P(f, q)=1$ indica que, para as instâncias nas que a estratégia não atingiu a melhor solução, encontrou soluções que estão, no máximo, dentro de um fator $2^{q}$ vezes a melhor solução (seja gap ou tempo) encontrada. A melhor estratégia é aquela que converge para $P(f, q)=1$ mais rapidamente. Os valores em negrito indicam as melhores estratégias em GAP e os valores sublinhados indicam as melhores estratégias em tempo computacional.

Para o modelo 1, observa-se na Tabela 1 que três estratégias (CPX8, CPX9 e CPX15) convergiram mais rapidamente no perfil de desempenho do gap, pois foram as que atingiram $P(f, q)=1$ com o menor valor $q$ (1.756). Em relação ao tempo computacional, as melhores estratégias foram CPX2, CPX7, CPX9 e CPX15 que atingiram $P(f, q)=1$ com valores $q$ em torno de 2.9. Porém, as estratégias CPX2 e CPX7 estão entre as piores no critério gap ( $q>7.4$ quando $P(f, q)=1$ ) e a estratégia CPX8 é dominada por CPX15 e CPX9 no critério de tempo computa- 
cional. Assim, CPX9 e CPX15 foram as que mostraram os melhores resultados levando em conta os dois critérios, gap e tempo.

Tabela 1: Valores extremos das curvas de perfis de desempenho

\begin{tabular}{|c|c|c|c|c|c|c|c|c|}
\hline \multirow{3}{*}{ CPXs } & \multicolumn{4}{|c|}{ Modelo 1} & \multicolumn{4}{|c|}{ Modelo 2} \\
\hline & \multicolumn{2}{|c|}{ GAP } & \multicolumn{2}{|c|}{ Tempo } & \multicolumn{2}{|c|}{ GAP } & \multicolumn{2}{|c|}{ Tempo } \\
\hline & $P(f, q)^{*}$ & $q * *$ & $P(f, q)^{*}$ & $q^{* *}$ & $P(f, q)^{*}$ & $q^{* *}$ & $P(f, q)^{*}$ & $q^{* *}$ \\
\hline CPX1 & 0.0833 & 7.802 & 0.0833 & 3.924 & 0.3333 & 5.007 & 0.5000 & 3.064 \\
\hline CPX2 & 0.1250 & 7.761 & $\underline{0.1667}$ & $\underline{2.939}$ & 0.5000 & 0.3513 & $\underline{0.3750}$ & $\underline{2.341}$ \\
\hline CPX3 & 0.1250 & 7.889 & 0.0833 & 4.303 & 0.3750 & 1.685 & 0.3750 & 4.983 \\
\hline CPX4 & 0.0417 & 7.761 & 0.1250 & 5.115 & 0.3333 & 6.872 & 0.3750 & 5.072 \\
\hline CPX5 & 0.0417 & 7.802 & 0.0833 & 3.750 & 0.3750 & 6.253 & 0.3750 & 4.524 \\
\hline CPX6 & 0.0417 & 7.761 & 0.2917 & 3.264 & 0.4167 & 0.3508 & 0.3750 & 4.630 \\
\hline CPX7 & 0.2500 & 7.457 & $\underline{0.1250}$ & $\underline{2.815}$ & 0.4583 & 0.9995 & 0.3750 & 7.079 \\
\hline CPX8 & 0.0833 & 1.756 & 0.1667 & 4.395 & 0.5000 & 0.5075 & 0.4167 & 8.288 \\
\hline CPX9 & 0.1667 & 1.756 & $\underline{0.0833}$ & $\underline{2.907}$ & 0.4167 & 0.5075 & 0.3750 & 5.894 \\
\hline CPX10 & 0.0417 & 7.802 & 0.0833 & 5.648 & 0.3333 & 0.6188 & $\underline{0.4167}$ & $\underline{2.439}$ \\
\hline CPX11 & 0.0417 & 7.802 & 0.2500 & 3.793 & 0.4167 & 0.3513 & $\underline{0.4167}$ & $\underline{2.239}$ \\
\hline CPX12 & 0.1250 & 7.802 & 0.0833 & 4.710 & 0.3333 & 7.501 & 0.3750 & 5.072 \\
\hline CPX13 & 0.0417 & 7.802 & 0.0833 & 4.823 & 0.3333 & 7.501 & 0.3750 & 5.072 \\
\hline CPX14 & 0.0833 & 7.802 & 0.1667 & 3.921 & 0.3333 & 5.007 & 0.4583 & 2.972 \\
\hline CPX15 & 0.1667 & 1.756 & $\underline{0.3333}$ & $\underline{2.833}$ & 0.5000 & 0.2868 & $\underline{0.6667}$ & $\underline{2.204}$ \\
\hline
\end{tabular}

Além disso, a estratégia CPX9 (CPX15) atingiu o melhor gap para 16,6\% (16,5\%) das instâncias e apresentou, para as demais instâncias, soluções que estão, no máximo, dentro de um fator $2^{1,75} \approx 3,4\left(2^{1,75} \approx 3,4\right)$ vezes o melhor gap obtido. É importante acrescentar que, tanto CPX9 quanto CPX15, atingiram soluções que estão a menos de $1 \%$ da solução ótima para todas as instâncias. Em relação ao tempo computacional, CPX9 (CPX15) atingiu o melhor tempo para 8,3\% (33,3\%) das instâncias e apresentou, para as demais instâncias, tempos computacionais dentro de um fator $2^{2,91} \approx 7,5\left(2^{2,83} \approx 7,1\right)$ vezes o melhor tempo obtido. Considerando que a estratégia CPX15 obteve resultados similares aos de CPX9 em gap e uma convergência mais rápida que CPX9 em tempo computacional, pode-se dizer que a estratégia CPX15 é a melhor estratégia CPLEX para o modelo 1.

A Tabela 2 resume os resultados das estratégias CPX1 (default) e CPX15 para o modelo 1. Apresentam-se os valores médios da função objetivo, do tempo computacional e do gap para cada grupo de instâncias. Note que existe um ganho significativo com a melhor estratégia CPLEX (CPX15) em relação à configuração default, tanto em tempo quanto em gap. 0 custo médio passou de 1.356.173.850 com CPX1 para 206.387.307 com CPX15, uma redução de 84,78\%. 0 tempo computacional médio reduziu de 1.290 segundos com CPX1 para 654 segundos com CPX15, uma diferença de, aproximadamente, 50\%. Para as instâncias pequenas, os resultados foram similares em tempo computacional e em relação ao custo da função objetivo. Para as instâncias médias, a melhoria foi significativa apenas em tempo computacional. Para as instâncias 
grandes, a melhoria foi em tempo e em custo da função objetivo. De fato, são as instâncias grandes as que mais influenciam na redução do custo total médio.

Tabela 2: Valores médios dos resultados das estratégias CPX1 e CPX15 para o modelo 1

\begin{tabular}{ccccccc}
\hline & \multicolumn{3}{c}{ CPX1 (default) } & & \multicolumn{3}{c}{ CPX15 } \\
Instâncias & Valor F.0 & $\begin{array}{c}\text { Tempo } \\
\text { (Seg.) }\end{array}$ & GAP (\%) & Valor F.O & $\begin{array}{c}\text { Tempo } \\
\text { (Seg.) }\end{array}$ & GAP (\%) \\
\hline Pequenas & 34.950 & 32,79 & 0,90 & 34.926 & 35,70 & 0,84 \\
Médias & 108.324 .706 & 237,50 & 0,30 & 108.675 .417 & 35,66 & 0,36 \\
Grandes & 3.960 .161 .895 & $3.600,00$ & 87,38 & 510.451 .578 & $1.891,00$ & 0,61 \\
Média & 1.356 .173 .850 & $1.290,10$ & 29,53 & 206.387 .307 & 654,12 & 0,60 \\
\hline
\end{tabular}

Para o modelo 2, a estratégia CPX15 é evidentemente a melhor pois convergência mais rapidamente em ambos os perfis de desempenho (ver Tabela 1), i.e., gap $(q=0,2868)$ e tempo computacional $(q=2,204)$. Embora CPX15 seja a melhor estratégia, outras estratégias como CPX2, CPX6 e CPX11 apresentaram também bons resultados em relação ao gap e estratégias como CPX2, CPX10 e CPX11 apresentaram bons resultados no que se refere a tempo computacional. CPX15 atingiu o menor gap para 50,0\% das instâncias e o menor tempo computacional para $66,67 \%$ das instâncias, e obteve soluções que estão dentro de um fator $2^{0,2868 \approx 1,2}$ e 22,204 $\approx 4,6$ vezes o melhor gap e tempo computacional obtido, respectivamente.

A Tabela 3 resume os resultados das estratégias CPX1(default) e CPX15 para o modelo 2. Em média, houve melhoria no tempo computacional das instâncias resolvidas com a estratégia CPX15. Porém, não houve melhoria significativa no custo total médio. 0 custo médio passou de 1.275.826.204 com CPX1 para 1.274.510.835 com CPX15, uma redução de $0,1 \%$. 0 tempo médio passou de 2.404 com CPX1 para 1.586 com CPX15, uma redução de 34,05\%. Para as instâncias pequenas não existiu melhoria com os ajustes nos parâmetros do solver CPLEX. Para as instâncias médias, a melhoria foi tanto em tempo computacional quanto em gap. Para as instâncias grandes, não houve melhoria em relação a nenhuma medida de desempenho.

Tabela 3: Valores médios dos resultados das estratégias CPX1 e CPX15 para o modelo 2

\begin{tabular}{ccccccc}
\hline & \multicolumn{3}{c}{ CPX1 (default) } & \multicolumn{3}{c}{ CPX15 } \\
Instâncias & Valor F.0 & $\begin{array}{c}\text { Tempo } \\
\text { (Seg.) }\end{array}$ & GAP (\%) & Valor F.0 & $\begin{array}{c}\text { Tempo } \\
\text { (Seg.) }\end{array}$ & GAP (\%) \\
\hline Pequenas & 40.674 & 12.83 & 0,6532 & 40.585 & 22,77 & 0,5639 \\
Médias & 25.814 .099 & $3.600,00$ & 21,60 & 21.868 .081 & 1.136 & 0,5057 \\
Grandes & 3.801 .623 .838 & $3.600,00$ & 99,55 & 3.801 .623 .838 & $3.600,00$ & 99,55 \\
Média & 1.275 .826 .204 & $2.404,28$ & 40,60 & 1.274 .510 .835 & $1.586,26$ & 33,54 \\
\hline
\end{tabular}

\subsection{Análise da solução da instância M1 para os modelos 1 e 2}

Nessa seção são analisados os resultados para a instância base M1 com ambos os modelos e da instância M3 com o modelo 1. As duas instâncias apresentam um gap de otimalidade menor do que $1 \%$. No modelo 1, considerou-se que para todas as instâncias existe um número de veículos suficientemente grande, exceto para a instância M3 na qual se limitou a quantidade de veículos 
disponíveis (35 caminhões, 15 barcos e 10 helicópteros). No modelo 2, por outro lado, a quantidade de veículos que podem ser utilizados está limitada de forma natural pela cardinalidade do conjunto L'. A tabela 4 apresenta os custos considerados na função objetivo e a Tabela 5 apresenta os resultados das principais decisões na solução das instâncias M1 e M3, tais como o número de veículos contratos etc.

Tabela 4: Custo parciais referentes às soluções das instâncias M1 e M3

\begin{tabular}{|c|c|c|c|c|c|c|c|c|c|}
\hline \multirow{4}{*}{ Mod. 1} & \multirow[b]{2}{*}{ Ins. } & \multicolumn{8}{|c|}{ Custos } \\
\hline & & Custo Total & $\begin{array}{c}\text { Abrir } \\
\text { centros }\end{array}$ & $\begin{array}{l}\text { Operar } \\
\text { Centros }\end{array}$ & $\begin{array}{c}\text { Contratar } \\
\text { veículos }\end{array}$ & Transporte & $\begin{array}{c}\text { Atender } \\
\text { Demanda }\end{array}$ & Estoque & Atraso \\
\hline & $\mathrm{M} 1$ & 17.286 .460 & 2.400 & 48.400 & 2.513 .996 & 96.922 & 28.468 & 35.567 & 14.560 .707 \\
\hline & M3 & 750.594 .039 & 2.000 & 42.000 & 1.390 .550 & 3.340 & 2.976 & 5.818 & 749.147.355 \\
\hline Mod. 2 & M1 & 15.875 .625 & 2.200 & 46.900 & 886.353 & 45.089 & 210.431 & 38.332 & 14.646 .320 \\
\hline
\end{tabular}

Tabela 5: Principais decisões apoiadas pelos modelos associadas às instâncias M1 e M3

\begin{tabular}{ccccccccc}
\hline & & \multicolumn{3}{c}{ Veículos contratados } & Centros & Produtos & $\begin{array}{c}\text { Produtos } \\
\text { atrasados }\end{array}$ & $\begin{array}{c}\text { Nível de } \\
\text { serviço } \\
\text { (\%) }\end{array}$ \\
\hline & Ins. & Caminhão & Barco & Helicóptero & abertos & estocados & atrasadon \\
\hline Mod. 1 & M1 & 7032 & 415 & 0 & 12 & 123.427 & 25.497 & 99,64 \\
Mod. 2 & M1 & 200 & 150 & 150 & 10 & 6.145 & 3.778 .748 & 12,98 \\
\hline
\end{tabular}

O custo total da solução da instância M1 no modelo 1 é 8.8\% maior do que no modelo 2 devido, principalmente, a uma redução nos custos de contratação de veículos e transporte quando é permitida a reutilização de veículos no modelo 2. Note que, no modelo 1, foram utilizados, no total, 6.405 mais veículos do que no modelo 2 para a instância M1, o que representa uma quantidade significativa. Do ponto de vista de custos, a diferença entre as penalidades por demanda não atendida em ambos os modelos é marginal. Embora a instância M1 tenha sido construída considerando-se um número suficientemente grande de veículos, quando a mesma é resolvida pelo modelo 2, o número de veículos que pode ser utilizado é naturalmente limitado na tentativa de reduzir a cardinalidade do conjunto L'. Por essa razão, aparentemente não há vantagem, nesse caso, de reutilizar a frota de veículos. Entretanto, quando a limitação do número de veículos é imposta, gerando a instância M3, é possível notar que o modelo 1 não resulta em uma solução efetiva, pois grande parte dos produtos emergenciais não conseguem ser entregues devido à escassez de veículos.

Outra diferença importante nas soluções dos modelos é que, quando existe uma disponibilidade de todos os veículos, não é atrativo para o modelo 1 contratar helicópteros porque são veículos com preço de contratação e/ou manutenção altos. Para o modelo 2, por outro lado, a contratação de helicópteros é custo-efetivo, pois os mesmos podem ser reutilizados várias vezes dentro do horizonte de tempo e, portanto, seu alto custo é diluído entre várias viagens.

O número de centros de auxílio instalados foi similar nas soluções de ambos os modelos, correspondendo a quase $50 \%$ do total dos centros de auxílio candidatos. A maior parte dos centros de auxílio foi instalada nas principais/maiores cidades, sendo 4 centros em Petrópolis, 3 centros em Teresópolis, 4 centros em Nova Friburgo e 1 centro em Trajano de Moraes, de acordo com a solução da instância M1 obtida com o modelo 2.

0 estoque de produtos nos centros de auxílio é motivado, em ambos os modelos, pelo aproveitamento da capacidade dos veículos, ou seja, são enviados produtos adicionais em um período de baixa demanda para estocá-los nos centros de auxílio na tentativa de atender demandas 
de períodos futuros. Como esperado, esse comportamento é mais frequente nas soluções encontradas pelo modelo 2 , devido à limitação da frota de veículos disponíveis e por conta da reutilização.

Do ponto de vista prático o modelo 1 pode ser aplicado em situações em que a quantidade de veículos disponíveis é grande o suficiente, ou seja, a quantidade de veículos não é uma limitação para a distribuição dos suprimentos. Nesses casos, o modelo 1 é muito mais fácil de ser resolvido e, do ponto de vista do atendimento às vítimas, retorna melhores soluções do que o modelo 2. Porém, em situações reais, frequentemente a frota é limitada, fazendo com que as soluções do modelo 1 sejam ruins em termos atendimento às vítimas. De fato, note que o nível de serviço da instância M3 é em torno de 13\% por conta da falta de veículos necessários para atender as vítimas a tempo. Nesse caso, o modelo 2 fornece soluções mais efetivas em relação ao atendimento dos afetados porque menos veículos podem transportar mais produtos, reduzindo o tempo de privação e, simultaneamente, o tamanho da frota (o que acarreta na redução dos custos logísticos).

Como exemplo dessa situação, observe que a solução do modelo 1 considera uma utilização média de 745 veículos por dia, ao passo que o modelo 2 atinge uma distribuição tão efetiva com apenas 104 veículos por dia, dos quais 83 são caminhões, 14 são barcos e 6 são helicópteros, em média. A expectativa é que o modelo 2 gere soluções com uma frota mais diversificada por conta da oportunidade de reutilizar o mesmo veículo em várias viagens. Uma frota diversificada é crucial para atingir regiões colapsadas no pós-desastre, e.g., regiões alagadas ou estradas bloqueadas por conta de deslizes de terra, aumentando as chances de promover assistência humanitária com tempo de privação baixo, o que significa salvar mais afetados.

A principal desvantagem do modelo 2 em aplicações reais é a dificuldade de gerar soluções factíveis de boa qualidade em um tempo computacional plausível. Por essa razão, trabalhos futuros envolvem a proposição de técnicas mais sofisticadas de resolução.

Em ambos os modelos, é possível ainda considerar outras situações práticas, como quando alguns centros de auxílio não possuem infraestrutura necessária para receber algum tipo de veículo, e.g., helicópteros ou aeronaves. Nesse caso, bastaria impedir o fluxo desses tipos de veículos específicos nos arcos entre os depósitos e tais centros de auxílio com a utilização do parâmetro binário $u_{i j l t}(\xi)$.

\section{CONCLUSÕES E PERSPECTIVAS FUTUROS}

Esse artigo apresentou dois modelos de programação estocástica inteira-mista para o problema integrado de localização de centros de auxílio e distribuição de suprimentos em operações de resposta a desastres. Diferentemente da maioria dos trabalhos da literatura, os modelos propostos integram decisões de localização, distribuição e dimensionamento de frota heterogênea em um contexto multi-período, multi-produto e com considerações de custos de contratação, reutilização de veículos e tempo de transporte. Além disso, os modelos também consideram as incertezas inerentes às situações de desastres via um conjunto discreto de cenários. Para resolver instâncias práticas, foram exploradas diversas estratégias do método branch-and-cut do solver comercial CPLEX 12.5.1. Os resultados mostraram que, em geral, a utilização do método simplex dual, a proibição da geração de planos de corte e o aumento da frequência de utilização da heurística RINS melhoram o desempenho do método de solução na resolução dos modelos propostos. Adicionalmente, os resultados indicam que o modelo 2 fornece soluções mais efetivas do ponto de vista prático, pois é possível contratar uma frota menor para o abastecimento 
das vítimas e/ou atender uma maior quantidade de afetados com a mesma frota. Porém, o modelo 2 exige métodos de solução mais eficientes, o que pode ser investigado nos próximos passos dessa pesquisa.

Cabe ressaltar que as soluções dos modelos de otimização não têm o intuito de serem aplicadas arbitrariamente em contextos de operações humanitárias. A ideia é implementar as decisões otimizadas com o apoio de especialistas (ONGs, policiais, bombeiros, etc.) e combinar com ferramentas de informação geográfica (GIS e GPS, por exemplo) em sistemas de fácil manipulação computacional para que, de fato, tanto os decisores quanto as equipes de trabalho possam ser beneficiados com as ferramentas matemáticas. Os modelos também podem ser usados nas fases de preparação para simular diversos cenários de desastres e promover planos contingenciais mais efetivos em cada caso, por exemplo. Por hora, entretanto, o objetivo principal foi mostrar que há potencial para o desenvolvimento e utilização de modelos matemáticos em situações de desastres, por conta da complexidade desses eventos e limitação geral de recursos, e que os modelos podem ser razoavelmente bem resolvidos em um tempo aceitável na prática. A aplicação em casos reais depende também de políticas públicas e do convencimento de certos órgãos (por exemplo, a Defesa Civil Brasileira) de que vale a pena investir em ferramentas objetivas de apoio à decisão.

\section{AGRADECIMENTOS}

0 primeiro autor agradece à bolsa CAPES/DS. 0 segundo autor é grato às bolsas de pesquisa FAPESP (processos 2013/083032 e 2015/26453-7) e CNPq (processos 470154/2013-6 e 306237/2014-8) pelo apoio financeiro. 0 terceiro autor agradece à bolsa de pesquisa CNPq (processo 312569/2013-0) e FAPESP (processo 2016/01860-1).

\section{REFERÊNCIAS}

Afshar, A., Haghani, A., (2012). Modeling integrated supply chain logistics in real-time large-scale disaster relief operations. Socio-Economic Planning Sciences, v. 46, n. 4, p. 327-338. DOI: 10.1016/j.seps.2011.12.003.

Altay, N., Green, W., (2006). OR/MS research in disaster operations management. European Journal of Operational Research, v. 175, n. 1, p. 475-493. DOI: 10.1016/j.ejor.2005.05.016.

Apte, A., (2009). Humanitarian Logistics: A New Field of Research and Action. Foundations and Trends in Technology, Information and Operations Management, v. 3, n. 1, p. 1-100. DOI: 10.1561/0200000014.

Bozorgi-Amiri, A., Jabalameli, M., Alinaghian, M., Heydari, M., (2011). A modified particle swarm optimization for disaster relief logistics under uncertain environment. The International Journal of Advanced Manufacturing Technology, v. 60, n. 1-4, p. 357-371. DOI: $10.1007 / \mathrm{s} 00170-011-3596-8$.

Bozorgi-Amiri, A., Jabalameli, M., Mirzapour Al-E-Hashem, S., (2013). A multi-objective robust stochastic programming model for disaster relief logistics under uncertainty. OR Spectrum, v. 35, n. 4, p. 905-933. DOI: 10.1007/s00291-011-0268-X.

Busch, A., Amorim, S., (2011). Tragédia da região serrana do Rio de Janeiro em 2011: procurando respostas. Rio de Janeiro: Coordenação-Geral de Pesquisa da Escola Nacional de Administração Pública (ENAP), v. 1, p. 1-20.

Chang, M., Tseng, Y., Chen, J., (2007). A scenario planning approach for the flood emergency logistics preparation problem under uncertainty. Transportation Research Part E: Logistics and Transportation Review, v. 43, n. 6, p. 737-754. DOI: 10.1016/j.tre.2006.10.013.

Danna, E., Rothberg, E., Pape, C., (2005). Exploring relaxation induced neighborhoods to improve MIP solutions. Mathematical Programming, v. 102, n. 1, p. 71-90. DOI: 10.1007/s10107-004-0518-7.

Dolan, E., Moré, J., (2002). Benchmarking optimization software with performance profiles. Mathematical Programming, v. 91, n. 2, p. 201-213. DOI: $10.1007 / \mathrm{s} 101070100263$.

Döyen, A., Aras, N., Barbaroso, G., (2012). A two-echelon stochastic facility location model for humanitarian relief logistics. Optimization letter, p. 1123-1145. DOI: 10.1007/s11590-011-0421-0.

Eshghi, K.; Larson, R. C. Disasters: lessons from the past 105 years. Disaster Prevention and Management, v. 17, n. 1, p. 62-82, 2008. DOI: 10.1108/09653560911003705.

EM-DAT., (2016). The international disaster database. Disponível em: <http://www.emdat.be/advanced_search/index.html>.

Ferreira, D., Morabito, R., Rangel, S., (2010). Relax and fix heuristics to solve one-stage one-machine lot-scheduling models for small-scale soft drink plants. Computers \& Operations Research, v. 37, n. 4, p. 684-691. D0I: 10.1016/j.cor.2009.06.007.

Galindo, G., Batta, R., (2013). Review of recent developments in OR/MS research in disaster operations management. European Journal of Operational Research, v. 230, n. 2, p. 201-211. DOI: 10.1016/j.ejor.2013.01.039. 
Hutter, F., Hoos, H., Leyton-Brown, K., (2009). ParamILS : An automatic algorithm configuration framework. Journal of Artificial Intelligence Research, v. 36, p. 267-306.

ILOG, (2011). ILOG CPLEX 12.1: user's manual and reference manuals.

Li, L., Jin, M., Zhang, L., (2011). Sheltering network planning and management with a case in the Gulf Coast region. International Journal of Production Economics, v. 131, n. 2, p. 431-440. DOI: 10.1016/j.ijpe.2010.12.013.

Lin, Y., Batta, R., Rogerson, P., Blatt, A., Flanigan, M., (2012). Location of temporary depots to facilitate relief operations after an earthquake. Socio-Economic Planning Sciences, v. 46, n. 2, p. 112-123. DOI: 10.1016/j.seps.2012.01.001.

Mete, H., Zabinsky, Z., (2010). Stochastic optimization of medical supply location and distribution in disaster management. International Journal of Production Economics, v. 126, n. 1, p. 76-84. DOI: 10.1016/j.ijpe.2009.10.004.

Moreno, A., Alem, D., Ferreira, D., (2015). Coordenação da localização, distribuição e dimensionamento de frota em situações de desastre. In: BRAZILIAN SYMPOSIUM ON OPERATIONS RESEARCH. Proceedings..., p. 1499-1509.

Nolz, P., Doerner, K., Gutjahr, W., Hartl, R., (2010). A Bi-objective Metaheuristic for Disaster Relief Operation Planning. Advances in Multi-Objective Nature Inspired Computing. Studies in Computational Intelligence, p. 167-187. DOI: 10.1007/978-3-642-11218-8_8.

Noyan, N., (2012). Risk-averse two-stage stochastic programming with an application to disaster management. Computers \& Operations Research, v. 39, n. 3, p. 541-559. DOI: 10.1016/j.cor.2011.03.017.

Ortuño, M. T., Cristóbal, P., Ferrer, J. et al, (2013). Decision Aid Models for Disaster Management and Emergencies., Atlantis Computational Intelligence Systems. v. 7, p. 17-45. DOI: 10.2991/978-94-91216-74-9.

Rath, S., Gutjahr, W., (2011). A math-heuristic for the warehouse location-routing problem in disaster relief. Computers \& Operations Research, v. 42, p. 1-15. DOI: 10.1016/j.cor.2011.07.016.

Rawls, C., Turnquist, M., (2010). Pre-positioning of emergency supplies for disaster response. Transportation Research Part B: Methodological, v. 44, n. 4, p. 521-534. DOI: 10.1016/j.trb.2009.08.003.

Rawls, C., Turnquist, M., (2012). Pre-positioning and dynamic delivery planning for short-term response following a natural disaster. Socio-Economic Planning Sciences, v. 46, n. 1, p. 46-54. DOI: 10.1016/j.seps.2011.10.002.

Salmeron, J., Apte, A., (2010). Stochastic Optimization for Natural Disaster Asset Prepositioning. Production and Operations Management, v. 19, n. 5, p. 561-574. DOI: 10.1111/j.1937-5956.2009.01119.x.

Shen, Z., (2007). Integrated supply chain design models: a survey and future research directions. Journal of Industrial and Management Optimization, v. 3, n. 1, p. 1-27. DOI: 10.3934/jimo.2007.3.1.

Song, R., He, S., Zhang, L., (2009). Optimum transit operations during the emergency evacuations. Journal of Transportation Systems Engineering and Information Technology, v. 9, n. 6, p. 154-160. DOI: 10.1016/S1570-6672(08)60096-3. 